\title{
p53, HER2 and tumor cell apoptosis correlate with clinical outcome after neoadjuvant bevacizumab plus chemotherapy in breast cancer
}

\author{
SHERRY X. YANG ${ }^{1}$, SETH M. STEINBERG ${ }^{2}$, DAT NGUYEN $^{1}$ and SANDRA M. SWAIN ${ }^{3}$ \\ ${ }^{1}$ National Clinical Target Validation Laboratory, Division of Cancer Treatment and Diagnosis, and \\ ${ }^{2}$ Biostatistics and Data Management Section, Center for Cancer Research, National Cancer Institute, \\ Bethesda, MD; ${ }^{3}$ Washington Cancer Institute, Washington Hospital Center, Washington DC, USA
}

Received November 4, 2010; Accepted December 20, 2010

DOI: $10.3892 /$ ijo.2011.966

\begin{abstract}
Bevacizumab, an antibody to vascular endothelial growth factor (VEGF), has been incorporated into chemotherapy regimens in the treatment of several cancer types including breast cancer. The aim of this study was to identify tumor and angiogenic factors that potentially associate with outcome. In a pilot trial, 21 patients with inflammatory breast cancer and locally advanced breast cancer received bevacizumab plus doxorubicin-docetaxel chemotherapy before surgery. Baseline p53, HER2, tumor apoptosis, Ki67, estrogen receptor (ER), VEGF-A, serum VEGF (sVEGF), VEGFR2Y951 and microvessel density (MVD) were prospectively designed and determined by immunohistochemistry and enzyme-linked immunosorbent assay. Hazard ratios (HR) and $95 \%$ confidence intervals for survival and progressionfree survival (PFS) were estimated using Cox proportional hazards analyses. With a median follow-up of 65.9 months, patients with low apoptosis or p53-negative tumors had significantly longer survival than those with high apoptosis or p53-positive tumors (median 61.5 vs. 20.2 months; HR 0.22; $\mathrm{p}=0.011$ for apoptosis and median 59.6 vs. 24.2 months; HR 0.27 ; $\mathrm{p}=0.016$ for $\mathrm{p} 53$ ). Low Ki67 versus high Ki67 exhibited a trend towards association with survival (median 57.1 vs. 17.3 months, HR 0.34, p=0.07). Patients with HER2negative tumors had significantly longer PFS than those with HER2-positive tumors (median 31.2 vs. 9.4 months; HR 0.23; p=0.03). ER, VEGF-A, sVEGF, VEGFR2-Y951 and MVD were not significantly associated with outcome. Our data suggest that baseline p53, apoptosis and HER2 are each significantly associated with outcome in patients who received bevacizumab plus chemotherapy.
\end{abstract}

Correspondence to: Dr Sherry X. Yang, National Clinical Target Validation Laboratory, Division of Cancer Treatment and Diagnosis, National Cancer Institute, National Institutes of Health, Bldg. 37/Rm 1048, Bethesda, MD 20892, USA

E-mail: xy32m@nih.gov

Key words: apoptosis, bevacizumab, breast cancer, HER2, Ki67, p53

\section{Introduction}

During the past decade, anti-angiogenesis therapy has evolved as one of the promising approaches in the treatment of cancer including breast cancer although the mechanisms of action are complex $(1,2)$. However, a significant fraction of patients did not benefit from anti-angiogenesis therapy and/or in combination with chemotherapy $(3,4)$. Therefore, identification of biomarkers that associate with or potentially predict benefit for appropriate selection of patients has become a key issue of anti-angiogenesis therapy and an intense area of clinical and translational research. Recently, four randomized phase III trials demonstrated that addition of bevacizumab, a humanized monoclonal antibody directed to all forms of vascular endothelial growth factor A (VEGF-A), to chemotherapy either as first-line (E2100, AVADO and RIBBON-1) or second-line (RIBBON-2) therapy prolong progression-free survival (PFS) and increase response rate in patients with metastatic breast cancer $(5,6)$. However, none of these trials have demonstrated survival benefit from the addition of bevacizumab to chemotherapy. This was confirmed by a recent meta-analysis of overall survival (OS) data from the three first-line studies in metastatic breast cancer (3). Thus it is imperative to develop and validate biomarkers that could associate with outcome, especially survival.

Apoptosis is a process of programmed cell death characteristic of cell morphology changes, chromatin condensation, DNA fragmentation and cell death (7). The process of apoptosis is controlled by a diverse range of cell signals including nutrient deprivation and hypoxia. The latter are largely dependent on the status of blood supply and tissue angiogenesis. Ki67 is a nuclear protein that is associated with the cellular proliferation, which is widely used as a proliferation marker, especially as a tumor proliferation marker (8). It is present in all active phases of the cell cycle including $G_{1}, S$, $G_{2}$, and mitosis, but is absent from the resting cells $\left(G_{0}\right.$ phase) (9). Levels of Ki67 are low in G1 and S phases and highest in mitosis. Low Ki67 as compared with high Ki67 has been found to be associated with longer disease-free survival (DFS) and OS in node-positive breast cancer after adjuvant chemotherapy (10). 
p53 is a nuclear transcription factor encoded by the TP53 gene located on the short arm of chromosome 17 (17p13.1) (11). It is a tumor suppressor which regulates the cell cycle and plays a critical role in the regulation of apoptosis, genetic stability, or inhibition of angiogenesis (12). Wild-type p53 protein has a short half-life with low intracellular levels. However, stabilization of p53 protein in the absence of a stimulus such as DNA damage is a hallmark of loss of function secondary to a mutation, or interaction with viral or cellular oncoproteins (13). p53-positive tumors versus p53-negative tumors were associated with poor prognosis in breast cancer (14-16). The dysfunction of p53 has been shown to contribute to angiogenic switch during tumorigenesis through the amplification of hypoxia-inducible factor 1 (HIF-1) dependent responses to hypoxia (17). Furthermore, p53-deficient animal models were less responsive than wild-type p53 models to anti-angiogenic and cytotoxic combination therapy (18). It is, therefore, important to evaluate p53 status in association with patient outcome after treatment with anti-angiogenic agents and/or in combination with chemotherapy.

Human epidermal growth factor receptor 2 (HER2), encoded by the ERBB2 gene, activates multiple cellular signaling pathways that are involved in cellular proliferation and survival, and increases VEGF protein synthesis (19). The latter is regulated via activation of the mTOR/p70S6K capdependent translation pathway in human breast cancer cells (20). Overexpression of HER2 has been shown to correlate with the increased angiogenesis and VEGF-A expression in cancer cells (21). However, no studies thus far have assessed HER2 status in association with clinical outcome after bevacizumab plus chemotherapy.

Twenty-one previously untreated patients with inflammatory breast cancer (IBC) and locally advanced breast cancer (LABC) were entered into a pilot trial at the National Cancer Institute (NCI, Bethesda, MD), and treated with neoadjuvant bevacizumab for one cycle, followed by six cycles of bevacizumab plus docetaxel-doxorubicin chemotherapy before surgery. Given the implications of p53, HER2, tumor apoptosis (TUNEL), proliferation (Ki67), estrogen receptor (ER), VEGF-A, sVEGF, VEGFR2 activation (VEGFR2Y951) and microvessel density (MVD) on tumor progression, angiogenesis, and/or responses to anti-angiogenic therapy as well as to chemotherapy, we designed and incorporated evaluation of these markers in the study protocol $(22,23)$. We hypothesized that some of these factors could be associated with the treatment outcome of patients who received bevacizumab plus chemotherapy. The objectives of this study were to assess the association of OS and PFS with baseline expression of p53, HER2, ER, VEGF-A, VEGFR2-Y951, tumor apoptosis, proliferation, sVEGF, and MVD, as well as age, grade, and TNM stage.

\section{Patients and methods}

Patients, tumor biopsy and treatment. Study design, patient enrollment, drug administration, and evaluation of clinical response and toxicity have been previously described in detail $(22,23)$. In brief, the study was approved by the Institutional Review Board of the NCI. Eligible patients signed informed consent which included tissue biopsy and research use of collected tissue in compliance with federal and institutional guidelines. Twenty patients with IBC and one with LABC were enrolled from October 2001 to August 2004. Tumor biopsies were taken by mammotome on the breast tumor area or by either 16 or 18 gauge needles on ipsilateral lymph nodes. The biopsies were immediately fixed in formalin and subsequently sent to the Laboratory of Pathology, National Institutes of Health Clinical Center, for paraffin-embedding and tumor confirmation. Patients were treated with one cycle of bevacizumab at $15 \mathrm{mg} / \mathrm{kg}$ followed by six cycles of bevacizumab plus doxorubicin at $50 \mathrm{mg} / \mathrm{m}^{2}$ and docetaxel at $75 \mathrm{mg} / \mathrm{m}^{2}$ every three weeks prior to surgery and/or locoreginal therapy. Patients further received eight cycles of bevacizumab after surgery and those with hormone receptor-positive tumors also received tamoxifen or an aromatase inhibitor treatment. No patients received trastuzumab therapy.

Immunohistochemistry and quantitative analysis. p53, Ki67, VEGF-A, VEGFR2-Y951 and MVD were examined on formalin-fixed, paraffin-embedded biopsy sections using a standard avidin-biotin-peroxidase complex indirect immunoperoxidase procedure, and quantitatively analyzed with the assistance of a digital imaging system as previously described $(22,24,25)$. Antibodies used were appropriately validated prior to their application to biopsy sections $(22,23)$. HER2 status and ER status were determined on the diagnostic biopsy sections by the Laboratory of Pathology. Tumor apoptosis was examined by the terminal deoxynucleotidyl transferase (TdT)-mediated dUTP nick-end labeling (TUNEL) assay; and sVEGF was measured by human VEGF enzyme-linked immunosorbent assay (ELISA) as previously described $(22,26)$. The cut-offs for HER2, ER and p53 have been described by us and others previously $(27,28)$, and the ones for Ki67 labeling index, apoptosis index, VEGF-A, sVEGF, and VEGFR29-Y951 were arbitrarily chosen near the median of each biomarker (Table I). In brief, $2+$ and $3+$ levels of HER2 expression relative to 0 and $1+$ were scored as positive. ER and p53 were scored as positive if $10 \%$ or greater of malignant nuclei were stained. p53 protein was detected by a mouse monoclonal antibody (clone DO7, Vector Laboratories, Inc., Burlingame, CA) that recognizes both mutant and wild-type forms of the protein in 1:50 dilution (29). Breast cancer cell line MDA-MB-231 (p53-mutant), and a colon cancer specimen that express p53 were utilized as positive controls. The isotype control mouse immunoglobulins were used as negative controls.

Statistical analysis. The probability of OS or PFS as a function of time was estimated in each of the biomarker subgroups by the Kaplan-Meier method. The statistical significance of the differences in the associated comparisons was determined using the log-rank test. Hazard ratios (HR) and 95\% confidence intervals (CI) were determined using Cox proportional hazards regression analysis. Follow-up on living patients was current as of July, 2008, and used to determine the duration of OS and PFS. OS was determined from the on-study date to the date of death from any cause or last follow-up. PFS was calculated from the on-study date to the date of disease progression; the follow-up was censored if the patients went off study for reasons other than progression. The factors 
Table I. Expression frequencies of baseline markers.

\begin{tabular}{|c|c|c|c|c|}
\hline \multirow{2}{*}{$\begin{array}{l}\text { Marker } \\
\text { p53 }\end{array}$} & \multirow{3}{*}{$\begin{array}{l}\text { Median (range) } \\
6.7(0-95.7)\end{array}$} & \multicolumn{2}{|c|}{ Cut-off } & \multirow{2}{*}{$\begin{array}{l}\text { No. }(\%) \\
11(52)\end{array}$} \\
\hline & & $\geq 10$ & Negative & \\
\hline & & & Positive & $10(48)$ \\
\hline \multirow[t]{2}{*}{ HER 2} & $\mathrm{~N} / \mathrm{A}$ & $\geq 2+$ & Positive & $12(57)$ \\
\hline & & & Negative & $9(43)$ \\
\hline \multirow[t]{2}{*}{ ER } & N/A & $\geq 10$ & Positive & $11(71)$ \\
\hline & & & Negative & $6(29)$ \\
\hline \multirow[t]{2}{*}{ VEGF-A } & $1.2(0-39.4)$ & $\geq 2$ & Low & $11(55)$ \\
\hline & & & High & $9(45)$ \\
\hline \multirow[t]{2}{*}{ sVEGF } & $66.3(11.03-464.3)$ & $\geq 70$ & Low & $11(58)$ \\
\hline & & & High & $8(42)$ \\
\hline \multirow[t]{2}{*}{ pVEGFR2-Y951 } & $21.78(0.77-76.76)$ & $>20$ & Low & $10(50)$ \\
\hline & & & High & $10(50)$ \\
\hline \multirow[t]{2}{*}{ MVD } & $239.3(1-441.6)$ & $\geq 200$ & Low & $9(45)$ \\
\hline & & & High & $11(55)$ \\
\hline \multirow[t]{2}{*}{ Ki67 } & $29.1(5.5-84)$ & $>30$ & Low & $13(65)$ \\
\hline & & & High & $7(35)$ \\
\hline \multirow[t]{2}{*}{ Apoptosis } & $1.5(0-12.8)$ & $>2$ & Low & $12(60)$ \\
\hline & & & High & $8(40)$ \\
\hline
\end{tabular}

ER, estrogen receptor; HER2, human epidermal growth factor receptor 2; MVD, microvessel density; N/A, not applicable; No., number; pVEGFR2, phosphorylated vascular endothelial growth factor receptor 2; sVEGF, serum vascular endothelial growth factor; VEGF-A, vascular endothelial growth factor $\mathrm{A}$.

which were associated with outcome, with a univariate $p$-value of approximately 0.10 or less, as well as age and stage, were subsequently evaluated for their joint association with OS or PFS by a multivariable Cox proportional hazards model. All patients had consistently high grade tumors. A backward selection algorithm was used to determine the parameters to include in the final model. All p-values were two-tailed, reported without adjustment for multiple compa-risons, and considered to be statistically significant at $\mathrm{p}<0.05$.

\section{Results}

Patients, follow-up, and expression of tumor and angiogenic markers. Patient characteristics have been described previously (22). The median OS was 65.9 months while the median PFS was 17.4 months. Table I lists the expression frequency of p53, HER2, Ki67, apoptosis, ER, VEGF-A, sVEGF, VEGFR2Y951 or MVD. Expression of p53, HER2 or ER was $48 \%$, $43 \%$ or $29 \%$, consistent with the previous findings in studies with breast cancer and IBC $(19,30,31)$. Baseline Ki67 proliferation index ranged from 5.5-84\% (median $29.1 \%$ ), and apoptosis index ranged from 0 to $12.8 \%$ (median $1.5 \%$ ).

Marker status and overall survival. In patients treated with bevacizumab and docetaxel-doxorubicin chemotherapy, the HRs for the likelihood of survival among patients with tumors containing low levels of apoptosis versus those containing high levels of apoptosis was 0.22 ( $\mathrm{p}=0.011$ by the log-rank test; Table II). The HR among patients with p53-negative tumors compared with p53-positive tumors was 0.27 ( $\mathrm{p}=0.016$; Table II). Patients with low baseline apoptosis or p53negative tumors had significantly longer OS than those with high baseline apoptosis or p53-positive tumors (median 61.5 vs. 20.2 months; median 59.6 vs. 24.2 months; Table II; Fig. 1A and B). Patients with low tumor Ki67 versus high Ki67 exhibited a trend towards association with a longer survival (median 57.1 vs. 17.3 months; $p=0.07$; Table II). In addition, OS in patients with stage IIIA and IIIB was significantly longer than those with stage IIIC and stage IV (median 55.8 vs. 14.5 months; $\mathrm{p}=0.04)$. However, HER2, VEGF-A, sVEGF, ER, MVD, and VEGFR2(Y951) as well as age were not significantly associated with OS (Table II). Ki67 and apoptosis remained in the multivariable Cox proportional hazards model after eliminating other factors by a backward selection algorithm. When considered jointly, the HRs for the likelihood of survival among patients with low tumor apoptosis and Ki67 compared with those with high tumor apoptosis and Ki67 were $0.075(\mathrm{p}=0.004)$ and $0.098(\mathrm{p}=0.008)$, respectively (Table III).

Next, we performed an exploratory subset analysis for survival comparing patients with p53-negative tumors to those with p53-positive tumors by stratifying according to HER2 status, given the well-established roles for HER2 and p53 in the regulation of tumor angiogenesis $(17,20)$. In patients 
Table II. Overall survival by univariate analysis.

\begin{tabular}{|c|c|c|c|}
\hline Variable & Months (median) & Hazard ratio $(95 \% \mathrm{CI})$ & P-value \\
\hline Age, years & & & 0.60 \\
\hline$\geq 50$ vs. $<50$ & 55.0 vs. 24.9 & $0.763(0.273-2.134)$ & \\
\hline Stage & & & 0.041 \\
\hline IIIA+IIIB vs. IIIC+IV & 55.8 vs. 14.5 & $0.351(0.124-0.995)$ & \\
\hline p53 & & & 0.016 \\
\hline Negative vs. positive & 59.6 vs. 24.2 & $0.270(0.087-0.837)$ & \\
\hline HER2 & & & 0.34 \\
\hline Negative vs. positive & 54.3 vs. 26.7 & $0.602(0.212-1.708)$ & \\
\hline ER & & & 0.47 \\
\hline Positive vs. negative & 45.3 vs. 34.1 & $0.655(0.207-2.076)$ & \\
\hline VEGF-A & & & 0.94 \\
\hline High vs. low & 55.4 vs. 31.8 & $0.956(0.324-2.825)$ & \\
\hline sVEGF & & & 0.25 \\
\hline High vs. low & 57.0 vs. 26.0 & $0.507(0.155-1.663)$ & \\
\hline pVEGFR2-Y951 & & & 0.39 \\
\hline High vs. low & 52.1 vs. 31.8 & $0.601(0.185-1.955)$ & \\
\hline MVD & & & 0.74 \\
\hline Low vs. high & 41.8 vs. 38.1 & $0.822(0.258-2.616)$ & \\
\hline Ki67 & & & 0.07 \\
\hline Low vs. high & 57.1 vs. 17.3 & $0.344(0.103-1.150)$ & \\
\hline Apoptosis & & & 0.011 \\
\hline Low vs. high & 61.5 vs. 20.2 & $0.219(0.061-0.781)$ & \\
\hline
\end{tabular}

ER, estrogen receptor; HER2, human epidermal growth factor receptor 2; MVD, microvessel density; No., number; pVEGFR2, phosphorylated vascular endothelial growth factor receptor 2; sVEGF, serum vascular endothelial growth factor; VEGF-A, vascular endothelial growth factor A.

Table III. OS and PFS by multivariable Cox proportional hazards regression analysis.a

\begin{tabular}{|c|c|c|c|}
\hline Endpoint/variable & Parameter estimate & Hazard ratio $(95 \% \mathrm{CI})$ & P-value \\
\hline \multicolumn{4}{|l|}{ OS } \\
\hline Apoptosis & -2.59 & $0.075(0.01-0.43)$ & 0.004 \\
\hline Ki67 & -2.33 & $0.098(0.02-0.55)$ & 0.008 \\
\hline \multicolumn{4}{|l|}{ PFS } \\
\hline HER2 & -2.43 & $0.09(0.01-0.57)$ & 0.01 \\
\hline Apoptosis & -1.52 & $0.22(0.05-0.96)$ & 0.04 \\
\hline
\end{tabular}

${ }^{a}$ Shown are the final models by backward selection. HER2, human epidermal growth factor receptor 2; OS, overall survival; PFS, progression-free survival.

with HER2-negative tumors, those with p53-negative tumors survived significantly longer than those with p53-positive tumors (median not reached vs. 20 months; p=0.017; Fig. 2A).
In contrast, among patients with HER2-positive tumors, OS was similar in both p53-negative tumors and p53-positive tumors (median 20.0 vs. 23.4 months; p=0.78; Fig. 2B). 

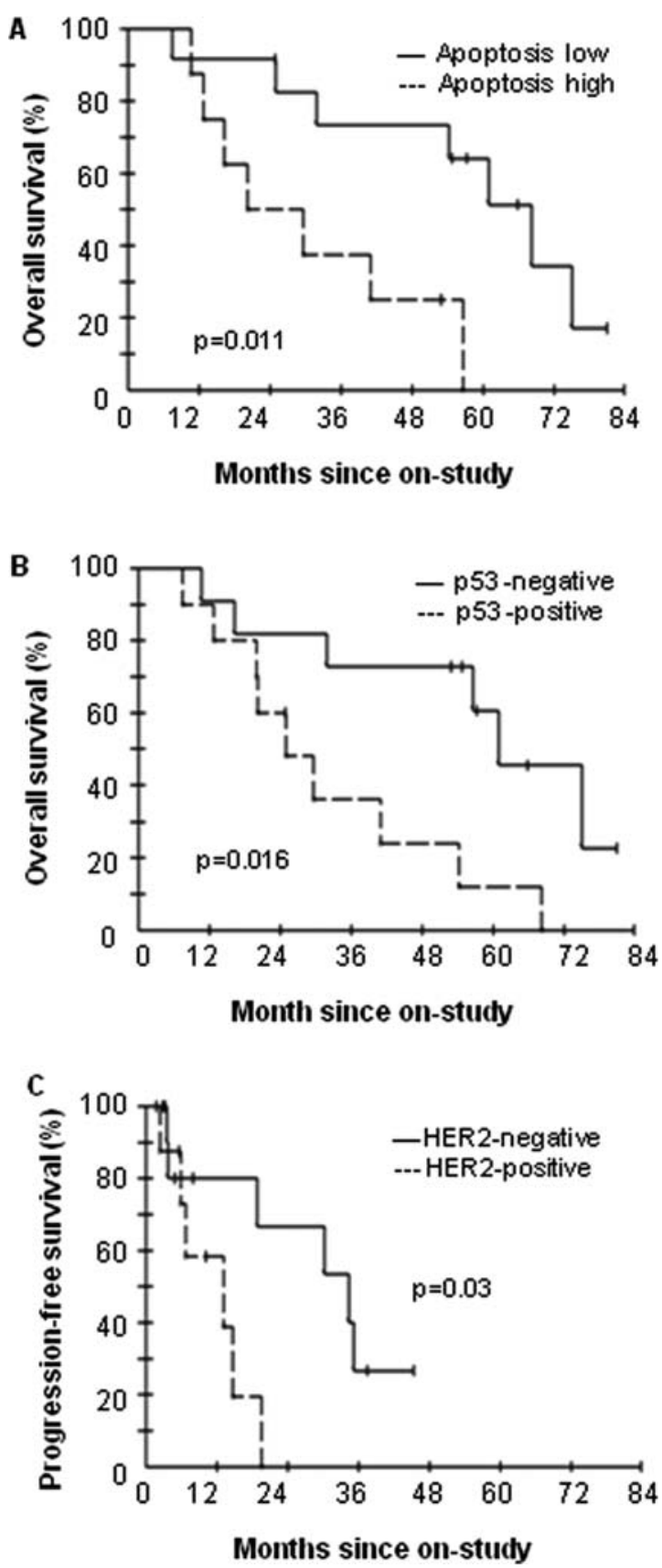

Figure 1. Overall survival comparing apoptosis-low group with apoptosishigh group (A) and p53-negative group with p53-positive group (B), or progression-free survival comparing HER2-negative group to HER2-positive group (C).

Marker status and progression-free survival. We also assessed the marker status in relationship to disease progression. Table IV shows the HRs for the risk of progression according to the status of markers. Patients with HER2-negative tumors had a significantly longer PFS than those with HER2positive tumors (median 31.2 vs. 9.4 months; $p=0.03$; Table III; Fig. 1C). The other angiogenic or tumor factors studied were not significantly associated with PFS by univariate analysis (Table IV). By multivariable Cox proportional hazards regression analysis using a backward selection algorithm, HER2 and apoptosis were significantly associated with progression when considered jointly in the model. The HRs for progression in HER2-negative tumors compared with HER2-positive tumors was $0.088(\mathrm{p}=0.01)$, and in low apoptosis versus high apoptosis was 0.22 ( $\mathrm{p}=0.04$; Table III).

\section{Discussion}

In this study, we investigated the relationships between tumor and angiogenic factors that play important roles in angiogenesis and tumor progression, and clinical outcome. Our results demonstrate that baseline p53 and apoptosis are significantly associated with OS by univariate analyses in patients who received neoadjuvant bevacizumab followed by bevacizumab plus doxorubicin-docetaxel chemotherapy. The data are substantiated by preclinical findings. p53 mediates inhibition of tumor angiogenesis through multiple mechanisms such as degradation of HIF-1 $\alpha$ (17), up-regulation of a collagen prolyl hydroxylase, which releases the antiangiogenic fragments of collagen type 4 and 18 to the extracellular matrix (32), and induction of microRNA-107 that inhibits HIF-1 (33). On the other hand, p53 dysfunction has been associated with the increase in VEGF expression and decrease in expression of thrombospondin-1, a potent inhibitor of angiogenesis or neovascularization (34). In addition, p53-deficient animal models were less responsive than wild-type p53 models to anti-angiogenic and cytotoxic combination therapy (18). In a large study, node-positive breast cancer patients received adjuvant fluorouracil-doxorubicin-cyclophosphamide or docetaxel-doxorubicin-cyclophosphamide chemotherapy; p53 was significantly associated with both DFS and OS for 1350 patients (16). In this pilot trial, p53 in significant association with survival may reflect the profound and collective effects of p53 on angiogenesis and responses to chemotherapy.

The low levels of baseline tumor apoptosis relative to high levels of baseline tumor apoptosis may represent a status of adequate versus inadequate tumor angiogenesis. The tumors with adequate angiogenesis status reflected by low levels of tumor apoptosis may be more sensitive to antiangiogenesis therapy, and thus was associated with outcome in patients after bevacizumab in combination with chemotherapy. Moreover, we found that baseline tumor apoptosis and proliferation are jointly and independently associated with the treatment outcome after multivariable modeling which potentially took into account of classical prognostic factors. Patients whose tumors with low intrinsic proliferation rate, possibly representative of a subset of patients with relatively indolent tumors, and baseline adequate tumor angiogenesis status (low levels of apoptosis index) identify subgroups of patients who had a significantly longer survival after bevacizumab anti-angiogenic therapy plus chemotherapy. In addition, patients with HER2-negative tumors had a significantly longer PFS than those with HER-positive tumors. HER2 and apoptosis are independently and jointly associated with disease progression as demonstrated by multivariable analysis. In previous preclinical and clinical studies, it has been shown that HER2 associates with disease progression, and increases angiogenesis in breast cancer $(21,35)$. The other markers examined were not significantly associated with PFS in this cohort.

However, there were no significant associations between some critical angiogenic factors (VEGF-A, sVEGF, VEGFR2- 

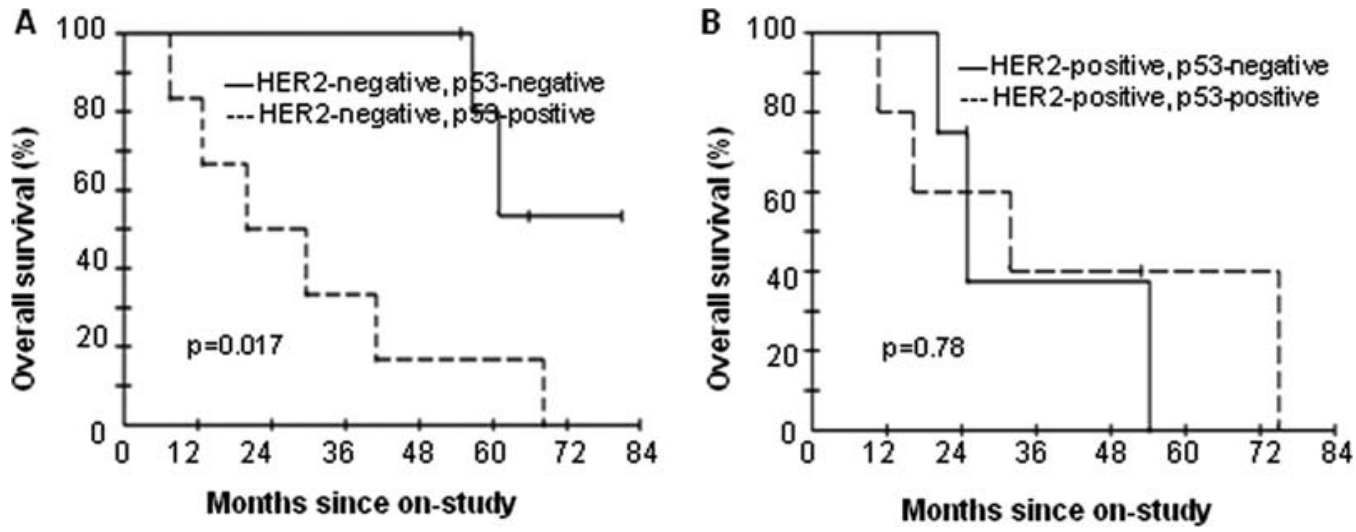

Figure 2. Overall survival (OS) in patients treated with bevacizumab plus chemotherapy according to HER2 and p53 status. OS comparing patients with p53negative tumors to those with p53-positive tumors in HER2-negative group (A) and HER2-positive group (B).

Table IV. Progression-free survival by univariate analysis.

\begin{tabular}{|c|c|c|c|}
\hline Variable & Months (median) & Hazard ratio $(95 \% \mathrm{CI})$ & P-value \\
\hline Age, years & & & 0.79 \\
\hline$\geq 50$ vs. $<50$ & 14.2 vs. 18.8 & $0.857(0.278-2.710)$ & \\
\hline Stage & & & 0.37 \\
\hline IIIA+IIIB vs. IIIC+IV & 19.0 vs. 6.4 & $0.561(0.156-2.014)$ & \\
\hline p53 & & & 0.36 \\
\hline Negative vs. positive & 21.0 vs. 16.1 & $0.586(0.185-1.858)$ & \\
\hline HER2 & & & 0.03 \\
\hline Negative vs. positive & 31.2 vs. 9.4 & $0.227(0.054-0.960)$ & \\
\hline ER & & & 0.13 \\
\hline Positive vs. negative & 30.2 vs. 13.7 & $0.232(0.030-1.811)$ & \\
\hline VEGF-A & & & 0.53 \\
\hline High vs. low & 16.3 vs. 18.3 & $0.682(0.204-2.283)$ & \\
\hline sVEGF & & & 0.95 \\
\hline High vs. low & 12.5 vs. 15.0 & $0.5961(0.279-3.305)$ & \\
\hline pVEGFR2-Y951 & & & 0.43 \\
\hline High vs. low & 24.0 vs. 14.3 & $0.620(0.186-2.071)$ & \\
\hline MVD & & & 0.80 \\
\hline Low vs. high & 16.8 vs. 16.2 & $0.854(0.246-2.964)$ & \\
\hline Ki67 & & & 0.67 \\
\hline Low vs. high & 22.7 vs. 11.2 & $0.767(0.222-2.642)$ & \\
\hline Apoptosis & & & 0.10 \\
\hline Low vs. high & 20.7 vs. 6.4 & $0.364(0.103-1.282)$ & \\
\hline
\end{tabular}

ER, estrogen receptor; HER2, human epidermal growth factor receptor 2; MVD, microvessel density; No., number; pVEGFR2, phosphorylated vascular endothelial growth factor receptor 2; sVEGF, serum vascular endothelial growth factor; VEGF-A, vascular endothelial growth factor A.

Y951 and MVD) and OS. Nor were these markers significantly associated with PFS. Instead, as discussed above, some critical tumor factors were found to be significantly associated with the treatment outcome.
Since bevacizumab was initially approved for use in HER2-negative metastatic breast cancer, and the facts that HER2 is associated with PFS and p53 is significant for OS by univariate analyses in this study, we performed an 
exploratory subset analysis of survival comparing patients with p53-negative tumors to those with p53-positive tumors by stratifying according to HER2 status. Interestingly, patients whose tumors were both HER2-negative and p53negative survived significantly longer than those whose tumors were HER2-negative and p53-positive after the combination treatment (Fig. 2A). p53 negativity, possibly functioning as a negative regulator of tumor angiogenesis and as a tumor suppressor, and the lack of HER2 overexpression, a positive regulator of angiogenesis and tumor progression, are critical for survival in patients who received bevacizumab plus chemotherapy. However, these data are considered to be hypothesis-generating due to the small sample size, and warrants further validation by other studies. In a Phase III study, the addition of bevacizumab to first-line irinotecan, 5-flurouracil, and leucovorin (IFL) prolonged median survival in patients with metastatic colorectal cancer (36). With a retrospective analysis of biomarker status in association with the treatment effect of bevacizumab, patients with p53 protein-negative tumors had a prolonged survival in patients treated with IFL plus bevacizumab as compared with those treated IFL plus placebo (median 25.07 vs. 16.26 months) (28).

Our results identified some potentially important markers which are associated with patient outcome after bevacizumab in combination with chemotherapy. However, the factors need to be investigated for their predictive roles in studies with larger sample sizes, especially in the context of randomized clinical trials in adjuvant, neoadjuvant and metastatic settings such as the National Surgical Adjuvant Breast and Bowel Project B40 and B46-I, and Eastern Cooperative Group E5103 and E2100. In these randomized studies, the biomarkers with regard to the treatment outcome with or without bevacizumab or treatment interactions can be assessed, and therefore their predictive value can be definitely determined.

\section{Acknowledgements}

This study was supported in part by the Division of Cancer Treatment and Diagnosis, and Center for Cancer Research, National Cancer Institute, National Institutes of Health. We thank Arlene Berman, Medical Oncology Branch, Center for Cancer Research, National Cancer Institute for patient followup. Dr S. Swain received research grants from Genentech and Bristol Myers Squibb, and travel supports from Genentech and Sanofi-Aventis to attend investigator meetings. She has been an uncompensated consultant for Genentech, Roche, and Sanofi-Aventis. The others have no potential conflict of interest.

\section{References}

1. Ferrara $\mathrm{N}$ and Kerbel RS: Angiogenesis as a therapeutic target. Nature 438: 967-974, 2005.

2. Jain RK: Lessons from multidisciplinary translational trials on anti-angiogenic therapy of cancer. Nat Rev Cancer 8: 309-316, 2008.

3. O'Shaughnessy J, Miles D, Gray RJ, et al: A meta-analysis of overall survival data from three randomized trials of bevacizumab (BV) and first-line chemotherapy as treatment for patients with metastatic breast cancer (MBC). J Clin Oncol 28 (Suppl.7): S7: abs. 1005, 2010.

4. Yang SX: Bevacizumab and breast cancer: current therapeutic progress and future perspectives. Expert Rev Anticancer Ther 9: 1715-1725, 2009.
5. Miller K, Wang M, Gralow J, et al: Paclitaxel plus bevacizumab versus paclitaxel alone for metastatic breast cancer. N Engl J Med 357: 2666-2676, 2007.

6. Miles DW, Chan A, Dirix LY, et al: Phase III study of bevacizumab plus docetaxel compared with placebo plus docetaxel for the first-line treatment of human epidermal growth factor receptor 2-negative metastatic breast cancer. J Clin Oncol 28: $3239-3247,2010$

7. Susin SA, Lorenzo HK, Zamzami N, et al: Molecular characterization of mitochondrial apoptosis-inducing factor. Nature 397: 441-446, 1999.

8. Schonk DM, Kuijpers HJ, van Drunen E, et al: Assignment of the gene(s) involved in the expression of the proliferationrelated Ki-67 antigen to human chromosome 10. Hum Genet 83: 297-299, 1989

9. Yerushalmi R, Woods R, Ravdin PM, Hayes MM and Gelmon KA: Ki67 in breast cancer: prognostic and predictive potential. Lancet Oncol 11: 174-183, 2010.

10. Hugh J, Hanson J, Cheang MC, et al: Breast cancer subtypes and response to docetaxel in node-positive breast cancer: use of an immunohistochemical definition in the BCIRG 001 trial. J Clin Oncol 27: 1168-1176, 2009.

11. Kern SE, Kinzler KW, Bruskin A, et al: Identification of p53 as a sequence-specific DNA-binding protein. Science 252: $1708-1711,1991$

12. $\mathrm{Xu} \mathrm{H}$ and el-Gewely MR: P53-responsive genes and the potential for cancer diagnostics and therapeutics development. Biotechnol Annu Rev 7: 131-164, 2001.

13. Blagosklonny MV: Loss of function and p53 protein stabilization. Oncogene 15: 1889-1893, 1997.

14. Silvestrini R, Benini E, Daidone MG, et al: p53 as an independent prognostic marker in lymph node-negative breast cancer patients. J Natl Cancer Inst 85: 965-970, 1993

15. Beenken SW, Grizzle WE, Crowe DR, et al: Molecular biomarkers for breast cancer prognosis: coexpression of c-erbB-2 and p53. Ann Surg 233: 630-638, 2001.

16. Dumontet C, Krajewska M, Treilleux I, et al: BCIRG 001 molecular analysis: Prognostic factors in node-positive breast cancer patients receiving adjuvant chemotherapy. Clin Cancer Res 16: 3988-3997, 2010

17. Ravi R, Mookerjee B, Bhujwalla ZM, et al: Regulation of tumor angiogenesis by $\mathrm{p} 53$-induced degradation of hypoxia-inducible factor 1alpha. Genes Dev 14: 34-44, 2000.

18. Yu JL, Rak JW, Coomber BL, Hicklin DJ and Kerbel RS: Effect of p53 status on tumor response to antiangiogenic therapy. Science 295: 1526-1528, 2002.

19. Slamon DJ, Godolphin W, Jones LA, et al: Studies of the HER-2/ neu proto-oncogene in human breast and ovarian cancer. Science 244: 707-712, 1989.

20. Klos KS, Wyszomierski SL, Sun M, et al: ErbB2 increases vascular endothelial growth factor protein synthesis via activation of mammalian target of rapamycin/p70S6K leading to increased angiogenesis and spontaneous metastasis of human breast cancer cells. Cancer Res 66: 2028-2037, 2006.

21. Kumar R and Yarmand-Bagheri R: The role of HER2 in angiogenesis. Semin Oncol 28: 27-32, 2001.

22. Wedam SB, Low JA, Yang SX, et al: Antiangiogenic and antitumor effects of bevacizumab in patients with inflammatory and locally advanced breast cancer. J Clin Oncol 24: 769-777, 2006.

23. Yang SX, Steinberg SM, Nguyen D, Wu TD, Modrusan Z and Swain SM: Gene expression profile and angiogenic marker correlates with response to neoadjuvant bevacizumab followed by bevacizumab plus chemotherapy in breast cancer. Clin Cancer Res 14: 5893-5899, 2008.

24. Tan AR, Yang X, Hewitt SM, et al: Evaluation of biologic end points and pharmacokinetics in patients with metastatic breast cancer after treatment with erlotinib, an epidermal growth factor receptor tyrosine kinase inhibitor. J Clin Oncol 22: 3080-3090, 2004.

25. Yang SX, Simon RM, Tan AR, Nguyen D and Swain SM: Gene expression patterns and profile changes pre- and post-erlotinib treatment in patients with metastatic breast cancer. Clin Cancer Res 11: 6226-6232, 2005.

26. Denduluri N, Yang SX, Berman AW, et al: Circulating biomarkers of bevacizumab activity in patients with breast cancer. Cancer Biol Ther 7: 15-20, 2008.

27. McCarthy NJ, Yang X, Linnoila IR, et al: Microvessel density, expression of estrogen receptor alpha, MIB-1, p53, and c-erbB2 in inflammatory breast cancer. Clin Cancer Res 8: 3857-3862, 2002. 
28. Ince WL, Jubb AM, Holden SN, et al: Association of K-ras, Braf, and p53 status with the treatment effect of bevacizumab. J Natl Cancer Inst 97: 981-989, 2005.

29. Turpeinen M, Serpi R, Rahkolin M and Vahakangas K Comparison of anti-p53 antibodies in immunoblotting. Biochem Biophys Res Commun 293: 850-856, 2002.

30. Hance KW, Anderson WF, Devesa SS, Young HA and Levine PH: Trends in inflammatory breast carcinoma incidence and survival: the surveillance, epidemiology, and end results program at the National Cancer Institute. J Natl Cancer Inst 97: 966-975, 2005.

31. Van den Eynden GG, Van der Auwera I, Van Laere S, et al: Validation of a tissue microarray to study differential protein expression in inflammatory and non-inflammatory breast cancer. Breast Cancer Res Treat 85: 13-22, 2004

32. Teodoro JG, Parker AE, Zhu X and Green MR: p53-mediated inhibition of angiogenesis through up-regulation of a collagen prolyl hydroxylase. Science 313: 968-971, 2006.
33. Yamakuchi M, Lotterman CD, Bao C, et al: P53-induced microRNA-107 inhibits HIF-1 and tumor angiogenesis. Proc Natl Acad Sci USA 107: 6334-6339, 2010.

34. Dameron KM, Volpert OV, Tainsky MA and Bouck N: Control of angiogenesis in fibroblasts by 553 regulation of thrombospondin-1. Science 265: 1582-1584, 1994.

35. Press MF, Bernstein L, Thomas PA, et al: HER-2/neu gene amplification characterized by fluorescence in situ hybridization: poor prognosis in node-negative breast carcinomas. J Clin Oncol 15: 2894-2904, 1997

36. Hurwitz H, Fehrenbacher L, Novotny W, et al: Bevacizumab plus irinotecan, fluorouracil, and leucovorin for metastatic colorectal cancer. N Engl J Med 350: 2335-2342, 2004. 